\title{
Pour une reconsidération des critères d'attractivité territoriale : Le cas des projets d'implantation de terminaux portuaires méthaniers au Québec
}

\author{
Yann Alix, École de Management de Normandie et \\ Emmanuel Guy, Université du Québec à Rimouski
}

\section{Introduction \\ Développement territorial et attractivité portuaire}

Les ports et les infrastructures terrestres afférentes ont toujours été des vecteurs du développement économique. L'attractivité portuaire sur le territoire se caractérise, entre autres, par l'implantation de capitaux, de ressources humaines aux compétences multiples et de structures pluri-industrielles. Les effets structurants se concrétisent par la densité d'une communauté portuaire élargie qui englobe des métiers aussi divers que l'avitaillement, les assurances ou encore la logistique de transports. Les activités portuaires intrinsèques démultiplient les retombées directes et indirectes sur un territoire qui s'étend aux confins de son arrière-pays ${ }^{1}$. En clair, un port, ses infrastructures et ses opérations soutiennent le développement économique local, régional, voire national et même international. Le rayonnement commercial des activités portuaires de Montréal dépasse largement l'aire d'influence de la grande métropole canadienne pour atteindre des aires industrielles comme Détroit, Chicago, voire même Kansas City ${ }^{2}$. Le grand port allemand de
Hambourg soutient l'émergence d'un réseau de plates-formes logistiques intérieures localisées à des centaines de kilomètres de ses quais. Shanghai, plus grand port du monde, rayonne bien au-delà des rives de la Mer de Chine avec l'alimentation de corridors logistiques de fret dans l'intérieur du continent chinois !

Un jeu complexe d'échelles territoriales se joue dorénavant dans l'appréciation des critères de l'attractivité des infrastructures portuaires. Les activités portuaires autogénèrent des activités connexes dédiées au navire et surtout à la marchandise. Elles peuvent se situer dans

l'arrière-cour immédiate du port comme à plusieurs dizaines de kilomètres. Plus un port est compétitif vis-à-vis de ses concurrents et plus son aire d'influence croît. Des cercles concentriques peuvent alors matérialiser le territoire sur lequel le port recrute et aussi depuis lequel des chargeurs industriels et des prestataires logistiques expédient leurs flux. Le dessin des zones d'influence met en perspective le recoupement des surfaces territoriales où les ports sont en concurrence pour attirer et fidéliser une clientèle par essence versatile (Figure 1). 
Figure 1

Interpénétration des aires de recrutement de clientèle : exemple de l'influence portuaire havraise face à ses concurrents nord et sud-européens

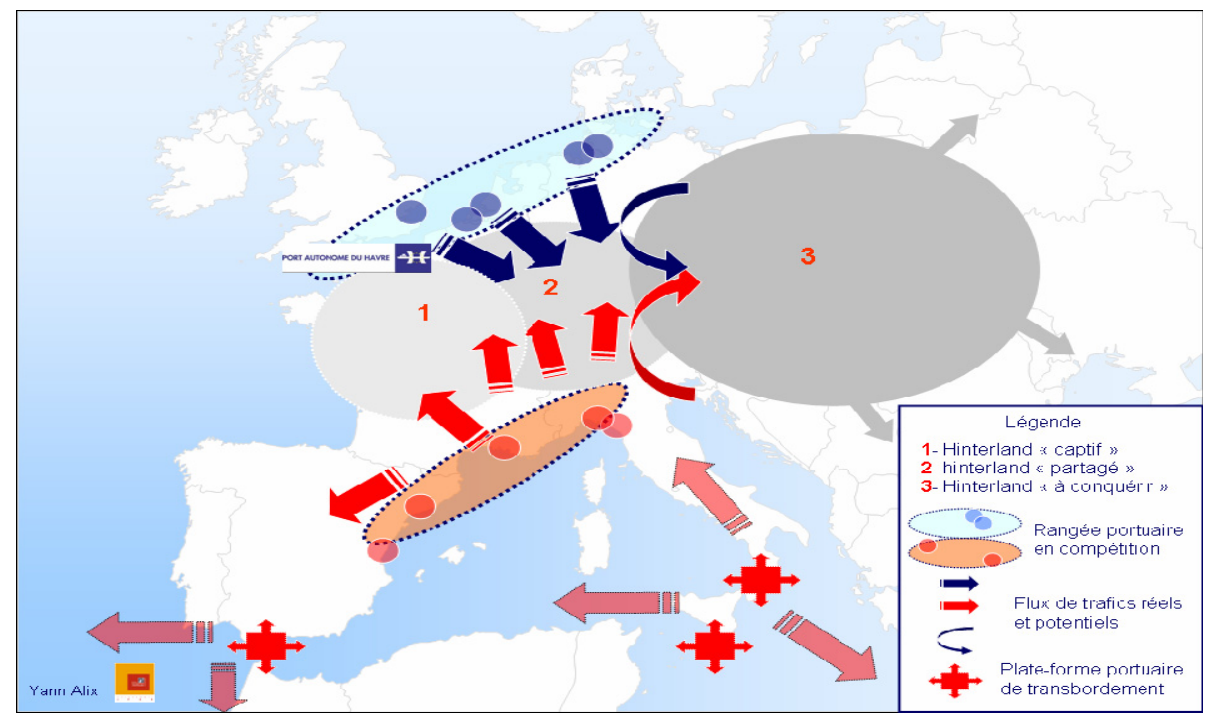

L'intensité de la maîtrise de l'arrière-pays est inversement proportionnelle à la distance physique qui sépare le port de ses concurrents immédiats. Sans être mathématique, cette règle met en avant le fait que le facteur distance est à prendre en compte dans l'intensité du rayonnement commercial d'un port. Bien sûr, les qualités des dessertes terrestres, la flexibilité des services modaux ou encore l'importance du maillage de plates-formes intérieures sont autant d'éléments qui viennent contrecarrer le binôme éloignement géographique/perte d'influence commerciale et logistique. La seule distance physique entre les terminaux portuaires et les marchés de l'hinterland peut être sublimée en fonction des rapports suivants :

- coûts totaux /distance ;

- temps final de transit/distance, et ;

- disponibilité / fiabilité des services modaux et logistiques par rapport à la distance ${ }^{3}$.
Toutefois, il n'en reste pas moins que le site, la situation, les réseaux de transport, la densité de population, le pouvoir économique ou encore la concentration industrielle et tertiaire demeurent les facteurs classiques de l'analyse de l'attractivité pour le développement d'une infrastructure portuaire.

D'autres facteurs prégnants tels que les aides fiscales, les facilités logistiques ou même les perspectives stratégiques de développements industriels peuvent être également cités (Figure 2). Enfin, les avantages concurrentiels que peuvent offrir un port et son territoire se déclinent à travers la disponibilité et le niveau de formation/qualification des personnels employables sur les futurs terminaux à investir. Tous ces éléments entrent dans une interaction dynamique et le poids respectif de chacun des paramètres se considère en fonction même de la nature du projet d'investissement portuaire. 


\section{Figure 2}

\begin{tabular}{|c|c|c|c|c|c|c|}
\hline & & Principales ca & gories dans la critériologie & e la localisation « tradit & nelle » des terminaux port & ires \\
\hline & & Géographiques & $\begin{array}{c}\text { Economiques et Démogra- } \\
\text { phiques }\end{array}$ & Logistiques & $\begin{array}{c}\text { Politiques \& institution- } \\
\text { nelles }\end{array}$ & Industrielles \\
\hline 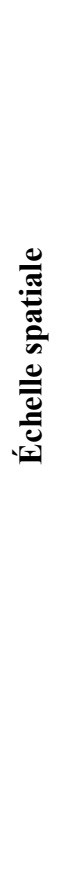 & $\begin{array}{l}\text { Méso - } \\
\text { Régionale/ } \\
\text { Nationale }\end{array}$ & $\begin{array}{l}\text { - Site \& situation } \\
\text { - Accessibilité nautique/terminal, } \\
\text { courants, conditions climatiques, } \\
\text { etc. } \\
\text { - Disponibilité de terrains et ré- } \\
\text { serves foncières } \\
\text { - Accessibilité terrestre et connec- } \\
\text { tivité aux réseaux de transport - } \\
\text { - Qualité et quantité des services } \\
\text { pluri-modaux } \\
\text { - Tarification des services } \\
\text { - Positionnement concurrentiel et } \\
\text { couverture compétitive de } \\
\text { l'hinterland partagé } \\
\text { - Proximité de ressources naturel- } \\
\text { les }\end{array}$ & $\begin{array}{l}\text { - Taille du marché de : } \\
\text { consommation/ } \\
\text { production/ } \\
\text { transformation } \\
\text { - Densité de population } \\
\text { \& Pouvoir d'achat } \\
\text { - Niveau de croissance et } \\
\text { perspectives à terme } \\
\text { - Réseaux bancaires } \\
\text { - Evaluation des solutions } \\
\text { alternatives (compétition } \\
\text { entre deux entités portuai- } \\
\text { res, voire entre deux termi- } \\
\text { naux sur un même port). }\end{array}$ & $\begin{array}{l}\text { - Plates-formes logisti- } \\
\text { ques sur zones dédiées } \\
\text { - Terrains et surfaces } \\
\text { disponibles } \\
\text { - Statut des terrains - } \\
\text { achat/location } \\
\text { - Employabilité de la } \\
\text { m.o. (qualifications et } \\
\text { spécialités) } \\
\text { - Expertises et synergies } \\
\text { logistiques }\end{array}$ & $\begin{array}{l}\text { - Impôts directs et indi- } \\
\text { rects } \\
\text { - Subventions et aides } \\
\text { fiscales } \\
\text { - Aménagement du terri- } \\
\text { toire et zones franches } \\
\text { - Orientation stratégique et } \\
\text { grands projets } \\
\text { d'infrastructures } \\
\text { (ex : Commission Euro- } \\
\text { péenne) } \\
\text { - Situation politique et } \\
\text { rivalités intestines (no- } \\
\text { tamment à l'échelle du } \\
\text { dvp régional). }\end{array}$ & $\begin{array}{l}\text { - Niveau de concentra- } \\
\text { tion industrielle et } \\
\text { manufacturière } \\
\text { - Spécialisation de } \\
\text { production en filières } \\
\text { (exemple de } \\
\text { l'automobile ou de } \\
\text { l'informatique) } \\
\text { - Projets de dvp indus- } \\
\text { triels } \\
\text { - Orientation des en- } \\
\text { treprises dans les } \\
\text { mouvements } \\
\text { d'imports/exports }\end{array}$ \\
\hline
\end{tabular}

Source : Alix, Guy et Pelletier, 2006 


\section{Pour une relecture de l'attractivité por- tuaire}

Dans le contexte de la présente contribution, les critères de la localisation stratégique d'un investissement portuaire sont revisités avec le cas particulier de l'implantation des terminaux méthaniers. La problématique repose sur le fait que de nouveaux paramètres prennent une importance grandissante dans les choix stratégiques de la localisation de l'investissement international. Les stratégies pour une implantation durable et rentable tendent à se complexifier avec le croisement d'intérêts divergents de catégories d'acteurs aux considérations différentes ${ }^{4}$.

En premier lieu, il convient de replacer les considérations de l'attractivité selon l'échelle spatiale. Cela signifie que l'appréciation de l'attractivité d'un projet portuaire se décompose selon que les retombées soient établies localement, nationalement ou même internationalement. Dans le cas des projets portuaires méthaniers, l'on se rend compte que les intérêts des principales catégories d'acteurs ne convergent pas ${ }^{5}$. Les citoyens installés dans le périmètre de la future implantation portuaire ne s'alignent pas nécessairement avec les stratégies des acteurs politiques et portuaires locaux/régionaux

Les pouvoirs publics interagissent différemment selon que l'on soit un élu local ou un responsable politique national. Les pouvoirs publics doivent composer entre le calcul des retombées directes et indirectes de l'implantation d'un projet portuaire et l'argumentaire d'un choix éminemment politique sur le développement énergétique national. La revitalisation d'espaces éloignés, l'aménagement du territoire ou encore la fixation d'activités industrialo-portuaires combinent les échelles spatiales de lecture des intérêts publics. Enfin, les logiques économiques, financières et territoriales des grands fournisseurs internationaux d'énergie se confrontent à l'impérieuse nécessité de faire valoir les dimensions locales/régionales dans leurs projets d'investissements (Figure 3).

Figure 3

Considérations de l'échelle spatiale des intérêts des principales catégories d'acteurs d'un projet portuaire méthanier

\begin{tabular}{|l|c|c|c|c|}
\hline \multirow{2}{*}{ Catégories d'acteurs } & \multicolumn{4}{|c|}{ Échelle spatiale des intérêts } \\
\cline { 2 - 5 } & Locale & Régionale & Nationale & Internationale \\
\hline Promoteurs énergétiques & + & + & ++ & +++ \\
\hline Pouvoirs publics & + & ++ & +++ & + \\
\hline Citoyens/consommateurs & +++ & ++ & + & + \\
\hline
\end{tabular}

Source : Alix, Guy et Pelletier, 2006

Ensuite, une nouvelle dimension informationnelle cultive un renouveau dans l'appréciation des critères de l'attractivité territoriale et dans l'acceptation sociétale des investissements. Les dimensions du risque environnemental, social, sociétal et technologique apparaissent dorénavant au cœur des débats. Ils s'ajoutent à tous les critères traditionnels d'évaluation de l'investissement dans les grands projets d'infrastructures. Ils peuvent même contrecarrer certaines perspectives économiques ou financières par l'apport de dimensions plus suggestives, relevant de questionnements citoyens jusqu'alors très peu élaborés dans l'histoire ancienne des constructions portuaires internationales.

Les groupes de pression et de citoyens ont les moyens d'un véritable argumentaire face aux dé- monstrations des industriels. Ils profitent de la nouvelle dimension consensuelle dans la phase amont de tous les grands projets d'infrastructures. Les débats et audiences publics sont systématisés et la plupart des projets ne peuvent voir le jour sans un consensus généralisé ${ }^{6}$. La consultation publique soutient la diffusion d'une information scientifique de qualité, débattue lors de séances où toutes les parties prenantes peuvent défendre leurs points de vue. Aspects sociétaux, culture environnementale, dimension écologique, perspectives sociales et sanitaires apparaissent comme autant de paramètres qui prennent une importance croissante dans les choix des industriels au moment de leurs investissements ${ }^{7}$. L'accès à l'information reste cependant à double tranchant avec le problème de l'évaluation et 
de l'interprétation. C'est le cas notamment avec la difficulté du consensus dans l'acceptation des niveaux de risques et le calcul des externalités pour les deux projets gaziers au Québec.

\section{Discussion sur le cas des projets de terminaux méthaniers au Québec}

À la fin 2005, pas moins de 7 projets portuaires méthaniers étaient enregistrés par l'Office National pour l'Énergie du Canada dont 5 concernaient l'Est du pays et 2 le Québec (Figure 4). Les projets Rabaska et Énergie Cacouna devraient s'implanter à moins de deux heures de route l'un de l'autre sur la rive sud du Saint-Laurent : le premier, en amont de la ville de Lévis et le second à Cacouna à proximité de Rivière-du-Loup. Ils ont comme vocation de répondre à la demande confirmée de gaz naturel des marchés québécois, ontariens et de l'Est des ÉtatsUnis. Tels que présentés dans la figure 5, les deux projets se développent en parallèle, soutenus par des groupes d'investisseurs distincts.

Aucun des terminaux projetés ne s'implante directement dans les grands ports canadiens à l'exception de Canaport à Saint-Jean, N-B. Le projet Rabaska propose une installation à l'intérieur des limites maritimes du Port de Québec, mais sur un site séparé des zones portuaires actuelles. Le point commun de tous ces projets est un certain l'éloignement des principales aires de concentration urbaine, économique ou industrielle. Cette caractéristique se traduit d'abord par le choix d'espaces indépendants des zones portuaires établies, invitant alors à rediscuter les paramètres classiques de l'attractivité territoriale.

Figure 4

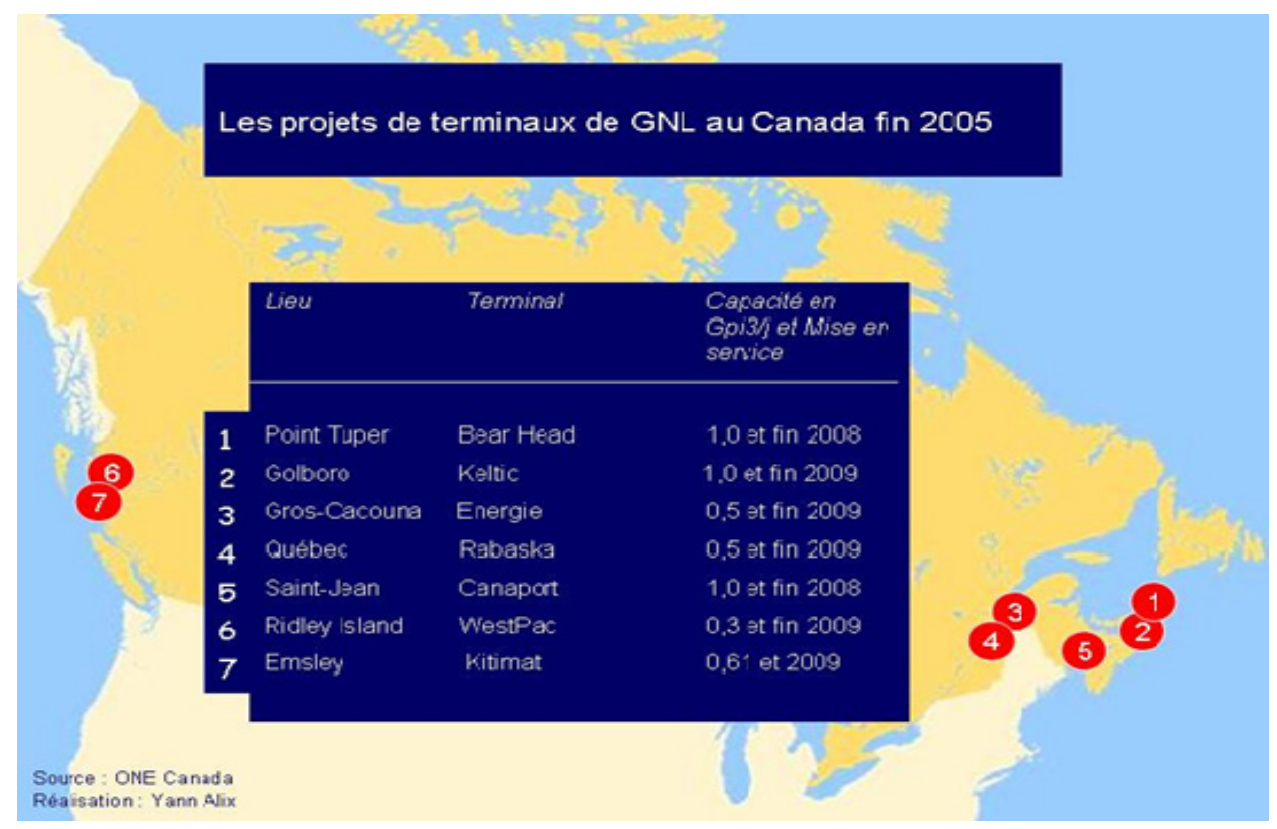

En premier lieu, il convient tout d'abord de rappeler que la localisation des récents projets portuaires méthaniers s'inscrit dans un contexte historique où les autorités états-uniennes ont favorisé, voire imposé, le remote siting dès $1970^{\circ}$. L'implantation des ports méthaniers se réalise sur des sites justement isolés des zones plus densément peuplées. Il serait tentant de voir là une mesure simple de gestion des risques d'incendie ou d'explosion liés à la manipulation du GNL. Mais les normes techniques, tant canadiennes qu'internationales, exigent des zones d'exclusion de l'ordre de quelques centaines de mètres et établissent des zones de risque négligeable dans un rayon souvent inférieur à $1 \mathrm{~km}$. Les distances invoquées ne sont que des ordres de grandeur puisque les normes n'imposent pas de distances fixes, mais proposent des formules de calcul de risques pour établir ces zones en tenant compte des particularités des installations et de leur environnement immédiat. Toutefois, l'opération de terminaux méthaniers ouest-européens dans des conditions de localisation finalement comparables à d'autres ter- 
minaux portuaires démontrent par ailleurs que la rupture avec les critères d'attractivité portuaires classiques ne peut s'expliquer que par des exigences techniques découlant de la nature du GNL. Les facteurs de différenciation semblent plutôt se trouver dans le développement de l'acceptabilité sociale de ces projets et plus encore dans la logique privée particulière dans laquelle ils s'insèrent.

Le secteur gazier n'apprécie pas les avantages logistiques et infrastructures d'un territoire de la même manière que l'industrie du conteneur ou le transport des marchandises générales diverses ${ }^{9}$. La connexion des terminaux gaziers à des usines de regazéification et des réseaux de pipelines soutient un développement souvent « intégré », tel un puzzle qui commence à la réception du navire et se termine à la distribution finale du gaz chez les consommateurs. Cette intégration verticale fait des installations portuaires des pièces d'équipements supplémentaires dans une chaîne logistique privée. Il s'agit d'un contraste marqué avec les dimensions

\section{Figure 5}

Quelques éléments chiffrés des deux projets portuaires méthaniers au Québec

\begin{tabular}{|l|c|c|}
\hline & Projet RABASKA & Projet ÉNERGIE CACOUNA \\
\hline Partenaires & $\begin{array}{c}\text { Gaz Metro } \\
\text { Enbridge Inc. } \\
\text { Gaz de France }\end{array}$ & $\begin{array}{c}\text { TransCanada } \\
\text { Pétro-Canada }\end{array}$ \\
\hline Coûts & $\begin{array}{c}775 \text { millions de CA\$ pour le termi- } \\
\text { nal }+65 \text { millions pour le gazoduc de } \\
42 \text { kilomètres }\end{array}$ & $\begin{array}{c}\text { Environ 700 millions de CA\$ + } \\
740 \text { millions de CA\$ pur le gazo- } \\
\text { duc de 240 km }\end{array}$ \\
\hline $\begin{array}{l}\text { Emplois directs + indirects (Phase } \\
\text { de construction) }\end{array}$ & $2440+2555$ & 4435 au total \\
\hline $\begin{array}{l}\text { Emplois directs }+ \text { indirects (Phase } \\
\text { d'exploitation) }\end{array}$ & $70+220$ & $35+105$ \\
\hline $\begin{array}{l}\text { Recettes gouvernementales totales } \\
\text { (Phase de construction) }\end{array}$ & $71+31$ millions de CA\$ \\
\hline $\begin{array}{l}\text { Recettes gouvernementales totales } \\
\text { (Phase d'exploitation) }\end{array}$ & $8,9+3,2$ millions de CA $\$$ & $17+12$ millions de CA\$ \\
\hline Mise en service programmée & Fin $2009 / 2010$ & $/$ \\
\hline
\end{tabular}

Source : Compilation des données de Rabaska et Énergie Cacouna et Gazoduc TQM

Le cas des terminaux portuaires méthaniers au Québec illustre combien les critères de choix s'établissent sur de nouveaux paramètres, ou tout du moins, redéfinissent l'importance relative de tous les paramètres à prendre en compte ${ }^{11}$. La relation, traditionnellement forte, entre un espace urbain et l'activité portuaire s'efface au profit d'espaces ruraux dotés de potentiels portuaires intéressants. Ou- multi-transporteurs, multi-expéditeurs, multiopérateurs qui caractérisent généralement les installations portuaires. Les paramètres de la décision dans le choix d'investissement sur un territoire donné s'en trouvent par conséquent modifiés, bouleversant les logiques traditionnelles de développement portuaire ${ }^{10}$. Cette dimension vient apporter un bémol au constat de la recherche d'isolement. En effet, si la localisation des projets méthaniers semble être en rupture avec le modèle d'une intégration spatiale étroite entre la ville et le port, il n'en demeure pas moins que les sites retenus ne correspondent pas du tout aux possibilités les plus isolées. Les cas québécois sont probants à cet égard: on a consenti à allonger, voire complexifier, les opérations maritimes pour pénétrer largement à l'intérieur de l'estuaire du Saint-Laurent. Les promoteurs ont, de toute évidence, accordé une grande importance aux possibilités de connexion au réseau de distribution par pipelines en place. 
tions. En principe, les insatisfactions face aux externalités telles que le bruit, le surplus de circulation, la pollution visuelle, l'interdiction d'accès à des zones côtières ou encore les risques d'accidents se gèrent d'autant mieux que les populations sont moins concentrées. De surcroît, les régions éloignées apparaissent plus souvent en manque de perspective économique à court, moyen et long terme et semblent possiblement moins mobilisables contre un investissement industrialo-portuaire d'envergure. Toutefois, les sites choisis dans les cas qui nous occupent appartiennent en fait à des régions qui ont connu des situations économiques plutôt intéressantes dans les dernières années, en particulier à $\mathrm{Ca}$ couna. Il serait simpliste de réduire la dynamique de l'acceptabilité sociale à des tentatives de contrôle des opposants. En effet, les retombées économiques à l'échelle locale/régionale sont concrètes et significatives : accès à une source d'énergie préalablement inaccessible, retombées fiscales intéressantes pour des municipalités et importante création d'emplois, en particulier durant la phase de construction. Ainsi, les réactions d'appuis ont été tout aussi spontanées que celles d'opposition à l'annonce des projets. Dans ce contexte, l'habileté à communiquer publiquement sa position apparait primordiale.

\section{Il apparaît nécessaire de revoir les critères classiques d'attractivité portuaire de façon à accorder plus d'importance aux logiques privées.}

Que ce soit le projet Rabaska ou le projet Énergie Cacouna, il est clair que les promoteurs ont largement anticipé ces nouvelles contraintes sociétales et environnementales. Les moyens alloués à la satisfaction de la revendication citoyenne illustrent combien les critères du choix d'implantation ont été parfaitement intégrés par les professionnels du secteur gazier. La gestion du consensus a été entretenue au fur et à mesure de la montée en puissance des mouvements de contestation. À travers le cadre formel des consultations du Bureau d'Audiences Publiques sur l'Environnement, la diffusion d'information par les promoteurs d'une part et l'articulation des préoccupations et oppositions des groupes environnementaux d'autre part ont permis la construction progressive d'un consensus positif de l'opinion public. Non pas que le tout se soit pas- sé sans heurts. Les projets soulèvent toujours l'opposition des groupes qui les ont mal reçus au départ et les promoteurs de Rabaska avaient dû déménager le projet vers son site actuel après avoir échoué à rallier une acceptation suffisante dans une municipalité voisine. Néanmoins, le projet Énergie Cacouna a maintenant franchi toutes les étapes de consultation et reçu l'approbation finale des autorités gouvernementales. La procédure se poursuit dans le cas du second projet, mais les médias dépeignent une acceptation tout de même majoritaire des citoyens. De notre point de vue, il est surtout intéressant de noter que ces deux grands projets portuaires ont en quelque sorte marqué définitivement une transformation des relations entre les contestataires locaux/régionaux et les promoteurs nationaux/internationaux. Ces derniers se doivent maintenant d'intégrer la dynamique du développement de l'acceptabilité sociale majoritaire comme une dimension à part entière de leurs projets. Ils deviennent proactifs à cet égard.

\section{Conclusion}

Les expériences vécues au Québec confirment que les promoteurs de projets méthaniers, sans abandonner la recherche des qualités portuaires naturelles ni l'accessibilité aux réseaux de distribution, favorisent une implantation sur des sites distincts. Ils investissent hors des zones portuaires traditionnelles et cherchent à se localiser en marge des concentrations urbaines et industrielles. Par conséquent, l'attractivité territoriale s'en trouve quelque peu bouleversée. Il apparaît nécessaire de revoir les critères classiques d'attractivité portuaire de façon à accorder plus d'importance aux logiques privées. C'est-à-dire considérer le port comme un équipement dans une ou des chaînes d'approvisionnement particulières, en même temps qu'un service public de soutient au développement de l'activité économique. Dans ce contexte, les perspectives d'acceptabilité sociale doivent également être formalisées comme critère à inclure dans la conceptualisation de l'attractivité portuaire.

\section{Notes et références}

1 Lim, Jung-Duk (2005). "Impact of Busan port and port related industries on regional economy ». Conference Proceedings. International Trade and Logistics, Corporate Strategies and the Global Economy. Faculté des Affaires Internationales, Sept. 28-29, 313-321. 
2 Guy, Emmanuel; ALIX, Yann (2007). “A Successful Upriver Port? Container Shipping in Montreal". Journal of Transport Geography, 15, 46-55

3 Alix, Yann (2006). «Construire une chaîne logistique de transport fiable : le rôle de l'autorité portuaire pour une desserte optimale des marchés enclavés ». Symposium international de l'AGPAOC. 26/28 juin. Niamey, Niger, 16p.

4 Hatem, F. (2006). L'investissement international en Europe. Rapport 2006. Agence Française pour les investissements internationaux. La Documentation Française. $142 \mathrm{p}$.

Alix, Yann ; GUY, Emmanuel ; Pelletier, Jean-François (2006). «Terminaux portuaires méthaniers : exception de la logique Ville-Port ou redéfinition d'une relation

Ruralité-Terminal? ». $10^{e}$ Conférence Internationale Villes et Ports. 5/9 novembre 2006. Sydney. Australie. $20 \mathrm{p}$.

6 Galichon, Pascal (2006), «Port 200 : Les dimensions essentielles du management de projet». Séminaire
Grands Projets. Executive MBA - Polytechnicum de Normandie. Le Havre, Mars 2006.

7 Scherrer, Paul (2006). « Port 2000, Le Havre. Le développement durable à grande échelle ». Séminaire Entrepreneurs Villes et Territoires. Ecole de Management de Paris. 5 Octobre 2005

8 Alix, Yann ; GUY, Emmanuel ; Pelletier, Jean-François (2006). Op. cit.

9 Carbone, Valentina ; DE MARTINO, Marcella (2003). « The changing role of ports in supply-chain management: an empirical analysis ». Maritime Policy and Management, Oct-Dec, Vol. 30, N4, 305-320.

10 Lacoste, R. (2006). Les opérateurs maritimes et portuaires européens dans la mutation de la chaîne de transport de marchandises en vrac. Essai de géographie économique. Thèse de doctorat. Université de Nantes. 399 p.

11 Coeck, Chris ; Haezendonck, Elvira ; Verbeke, Alain (1999). « The Introduction of an Ecological Dimension in Product Portfolio Analysis ». Proceedings of $8^{\text {th }}$ World Conference on Transport Research, Vol 4 : Transport Policy, 169-182. 